International Journal of Theoretical Physics, Vol. 33, No. 2, 1994

\title{
Dispersion, Topological Scattering, and Self-Interference in Multiply Connected Robertson-Walker Cosmologies
}

\author{
Roman Tomaschitz ${ }^{1}$ \\ Received March 2, 1993
}

\begin{abstract}
We investigate scattering effects in open Robertson-Walker cosmologies whose spacelike slices are multiply connected hyperbolic manifolds. We work out an example in which the 3-space is infinite and has the topology of a solid torus. The world-lines in these cosmologies are unstable, and classical probability densities evolving under the horospherical geodesic flow show dispersion, as do the densities of scalar wave packets. The rate of dispersion depends crucially on the expansion factor, and we calculate the time evolution of their widths. We find that the cosmic expansion can confine dispersion: The diameter of the domain of chaoticity in the 3-manifold provides the natural, time-dependent length unit in an infinite, multiply connected universe. In a toroidal 3-space manifold this diameter is just the length of the limit cycle. On this scale we find that the densities take a finite limit width in the late stage of the expansion. In the early stage classical densities and conformally coupled fields approach likewise a finite width; nonconformally coupled fields disperse. Self-interference occurs if the dispersion on the above scale is sufficiently large, so that the wave packet can overlap with itself. Signals can be backscattered through the topology of 3-space, and we calculate their recurrence times.
\end{abstract}

\section{INTRODUCTION}

In this paper we deal with the topological structure of space-time, in particular with the bearing of the topology on the microscopic motion, on the global behavior of the world-lines, and on the dynamics of wave packets.

We assume that the metric tensor of space-time can locally be described by a Robertson-Walker (RW) line element, and that the 3-space is infinite and has negative curvature.

'Theoretical Physics Group, Tata Institute of Fundamental Research, Bombay 400005 , India, and Physics Department, University of the Witwatersrand, Johannesburg, WITS 2050, South Africa. 
In Tomaschitz (1993a) we compared in the simply connected topology of the Minkowski hyperboloid the dispersion of classical densities that arises because of the instability of the flow lines with the dispersion of quantum mechanical wave packets. We found asymptotic equivalence of the classical and quantum dispersion in regimes where no annihilation/production processes occur. This is an important result, because it shows that in these universes the classical instability is well capable of producing the same dispersion phenomena as wave fields.

As was pointed out at length in the preceding papers (Tomaschitz, $1992 a-c$ ), a further peculiarity of open RW cosmologies of negative spatial curvature is that the spacelike slices can take a variety of topologies, which can even change in time.

If the 3-space is multiply connected and infinite, it is metrically deformable; its metric can vary in time even if its Gaussian curvature stays constant, in sharp contrast to the rigidity of the simply connected RW cosmologies with $S^{3}, \mathbb{R}^{3}, H^{3}$ as spacelike slices.

Another striking topological effect is the appearance of regions of chaoticity, and of bound-state wave fields localized on them (Tomaschitz, 1991).

In this paper we study topological scattering phenomena arising because of the multiple connectivity of the 3-space. Properly speaking, if a signal is emitted at some time, then a fraction of it may come back at later instants from different directions. This backscattering is somewhat reminiscent of the scattering that wave packets undergo in a metric that is rapidly varying in time (Schrödinger, 1939, 1956).

In Section 2 we study horospherical geodesic flows in a multiply connected 3-space manifold. Such a flow consists of bundles of parallel geodesics issuing from the boundary at infinity of the 3-space. We study probability densities evolving under this flow, and we derive a continuity equation for the 4-current density.

In Section 3 we compare this current with the current of wave fields satisfying the Klein-Gordon equation. In the case of the Minkowski hyperboloid as 3-space we found (Tomaschitz, 1993a) asymptotic equivalence of the classical current and the quantum current in regimes where we can identify positive- and negative-frequency solutions, for example, when the expansion factor is slowly varying. If the 3-space is multiply connected, this equivalence can be destroyed by topological scattering and the arising self-interference of the wave packet.

In Section 4 we make some comments on the topologies we have in mind, and sketch the simplest example of a hyperbolic 3-manifold that is open and multiply connected, namely that of a solid torus. 
In Section 5 we study topological scattering in RW cosmologies whose spacelike slices are solid tori. We will here again recognize the central part that the convex hull of the limit set of the covering group plays (Tomaschitz, 1992a). Its projection into the torus gives the limit cycle, a closed loop from which particles are spiraling out in infinite cycles. We study the scattering and dispersion of horospherical flows in the early and late stages of the expansion; in the quantum case we study self-interference. The expansion of 3-space provides a mechanism to confine dispersion. In fact, if we measure the widths of the densities in units of the circumference of the expanding limit cycle, we have the surprising phenomenon that wave packets as well as classical densities have finite limit widths. For further discussion on that we refer to the conclusion, Section 6.

\section{HOROSPHERICAL FLOWS IN RW COSMOLOGIES: THE GENERAL SETTING}

In Tomaschitz (1993a) we introduced the concept of a horospherical flow in a simply connected RW cosmology of negative spatial curvature, i.e., with Minkowski hyperboloids as spacelike slices. We constructed a 4-current $j_{c}^{\mu}$, a continuity equation, and an invariant measure. The full power of horospherical flows appears in the context of RW cosmologies whose spacelike slices are multiply connected hyperbolic 3-manifolds.

We formulate in this paper the concept of a horospherical flow on a multiply connected space-time manifold with the line element

$$
d s^{2}=-c^{2} d \tau^{2}+a^{2}(\tau) d \sigma^{2}
$$

Here $d \sigma^{2}$ denotes the line element of the open 3-space manifold, which we represent as a fundamental polyhedron $F$ in the Poincare ball $B^{3}$, or in the half-space model $H^{3}$ of hyperbolic space. The discrete group generated by the identifying transformations of the polyhedral faces we denote by $\Gamma$. For more details we refer to Tomaschitz (1992a).

We start with the continuity equation in $\mathbb{R}^{(+)} \times B^{3}$, which projects as it stands onto the manifold $\mathbb{R}^{(+)} \times F$, because of the invariance of $d \sigma^{2}$ under the Lorentz group, of which $\Gamma$ is a discrete subgroup:

$$
\frac{1}{\sqrt{-g}} \frac{\partial}{\partial x^{\mu}}\left(\sqrt{-g} j^{\mu}\right)=0
$$

Similar to the wave equation, we get solutions of (2.2) on the manifold $\mathbb{R}^{(+)} \times F$ by periodizing (method of images) with the covering group $\Gamma$ a solution $j_{B^{3}}^{\mu}$ in $B^{3}$. In fact we get all solutions in this way.

Let $x^{\mu}=(\tau, \mathbf{x}), \mathbf{x} \in B^{3}$, and $\gamma \in S O^{+}(3,1)$, the invariance group of $B^{3}$. We consider coordinate transformations of the kind $\tau^{\prime}=\tau, \mathbf{x}^{\prime}=\gamma^{-1} \mathbf{x}$, 
pp. 356-73 in attached files 
[cf. (3.4), (3.13)], we obtain equation (5.1), provided

$$
f_{, s} \sim A, \quad 2 \beta^{2} B^{2} \sim \alpha^{2} f_{, s s}^{2}
$$

which is just the condition for the asymptotic equivalence of $\rho_{c}$ [cf. (2.10)] and $\rho_{q}$ [cf. (3.12)] in the covering space. Condition (5.23) holds true, for example, in the case of adiabatic expansion, and for the expansion factors in (5.13) $-(5.15)$.

What is now the difference between (5.1) and (5.19)? What do the off-diagonal elements in $\Theta \Theta$ effect? First, $\psi^{\mathrm{r}}$ is constant on the horospherical projections in Figs. $4 \mathrm{a}$ and $4 \mathrm{~b}$ which are the wavefronts of $\psi^{\Gamma}$ in $\mathbb{R}^{(+)} \times F$. Concerning the $\eta$ averaging of (3.8), the same holds true as in the foregoing classical case: it effects the exponential decay of $\psi^{\Gamma}$ along the horospherical projections. Analogously to equation (5.17), we have

$$
f_{, s}\left(\tau+\Delta \tau_{n}\right)-f_{, s}(\tau)=n \log |K|
$$

Let us now assume as in (5.16) that the width of the Gaussian in (5.19) is much smaller than the length of the limit cycle. As long as $\operatorname{Re}\left(\lambda_{q}\right) \gg 1$ [cf. Section 6) the $|\Theta|^{2}$ in (5.19) is negligible, and there is then no difference from the classical case. But when $f_{, s s}^{2}$ increases, so that the width gets comparable with $R a(\tau) \log |K|$, then the fringes of the Gaussian in (5.18) start to overlap, and $\Theta \Theta$ in $(5.19)$ is no longer negligible, since $\operatorname{Re}\left(\lambda_{q}\right) \approx 1$. Because of the phases in the off-diagonal elements of $\Theta \Theta$, new interference peaks appear in the distribution $\rho_{q}^{\Gamma}$. Also note that $\operatorname{Re}\left(\pi^{2} / \lambda_{q}\right)$ is independent of $f_{\text {ss }}$, and $\rho_{q}^{\Gamma}$ in (5.22) will not approach a uniform distribution on the limit cycle for $f_{, s s}^{2} \rightarrow \infty$, contrary to the classical case $B^{2} \rightarrow \infty$, discussed after (5.16).

\section{CONCLUSION AND OUTLOOK}

There is a natural length scale in universes of multiple spatial connectivity, provided by the hyperbolic diameter $d(C(\Lambda) \backslash \Gamma)$ of the convex hull of the limit set. In our example in Section 5 we have

$$
d\left(C(\Lambda) \backslash \Gamma_{K}\right)=a(\tau) R \log |K|
$$

which is clearly the length of the limit cycle. This cycle can be very small, depending on the choice of $K$. A wave packet dispersing in the vicinity of such a tiny loop can easily start to overlap and interfere with itself.

The mean wavelength of the packet in (5.18) is

$$
\lambda_{0}=\frac{2 \pi}{s_{0}} R a(\tau)
$$

Its width is

$$
\chi=R a(\tau) \alpha^{-1}\left[\left(1+\alpha^{4} f_{, s s}^{2}\right)\right]^{1 / 2}
$$


One may take $\alpha=\sqrt{s_{0}}$. If the curvature of 3-space is very small, then $s_{0}$ must be very large to obtain a moderate $\lambda_{0}$, and therefore the averaging takes place over a very narrow interval of wavelengths, $\Delta \lambda=s_{0}^{-1 / 2} \lambda_{0}$. Likewise we can assume $\alpha \log |K| \gg 1$ [cf. (6.5)].

In the case of linear expansion $a(\tau) \sim \Lambda \tau$, we have (Tomaschitz, 1992c)

$$
f=\frac{m c^{2}}{\hbar} \tau-\frac{\left(1 / 4+s_{0}^{2}\right) \hbar}{2 m c^{2} \tau}+O\left(\tau^{-3}\right), \quad f_{m=0} \sim s_{0} \log \Lambda \tau
$$

and with (6.1), (6.3),

$$
\frac{\chi}{d}=\frac{1}{\alpha \log |K|}\left[1+\frac{\alpha^{4}}{2} \frac{\hbar^{2}}{m^{2} c^{4} \tau^{2}}+O\left(\tau^{-4}\right)\right], \quad\left(\frac{\chi}{d}\right)_{m=0} \sim \frac{1}{\alpha \log |K|}
$$

Thus the packet approaches a finite limit width on the scale of the expanding limit cycle $(6.1) ; \chi / d$ is here even decreasing. Whether a packet broadens or contracts depends on the choice of the phase of the wave function, but the $d$-scaled limit width is always finite under linear expansion. The $f$ in (6.4) is determined only up to a $\tau$-independent constant. We have chosen $f$ so that (5.23) is satisfied with $\tau_{0}=\infty$ in (2.11). If we choose the classical initial distribution at a finite $\tau_{0}$, then we have to add a time-independent constant to $f$ to achieve (5.23), and then the width broadens.

Even in the case $m=0$, when the wave packet is moving with the speed of light, we have a finite limit width relative to the limit cycle. In particular there will be no self-interference, in striking contrast to the static case $a(\tau)=1, f=\left(s_{0}^{2}+R^{2} m^{2} c^{2} / \hbar^{2}\right)^{1 / 2} \Lambda \tau$, and $\chi / d \rightarrow \infty$.

Remark. We study here the dispersion orthogonal to the horospherical wavefronts; cf. the comments after (5.16). If we take a square-integrable wave packet, it will also disperse along the horospherical projections in $F$. However, this dispersion orthogonal to the direction of propagation will be certainly even smaller.

For the frequencies we have from (6.4)

$$
v=\frac{m c^{2}}{2 \pi \hbar}+\frac{\left(s_{0}^{2}+1 / 4\right) \hbar}{4 \pi m c^{2}} \frac{1}{\tau^{2}}+O\left(\tau^{-4}\right), \quad v_{m=0} \sim \frac{s_{0}}{2 \pi \tau}
$$

Finally we discuss the recurrence times of the densities on the limit cycle $C(\Lambda) \backslash \Gamma_{K}$ [cf. (5.24)]. We study this again in the context of packets that are infinitely extended orthogonal to their direction of propagation (cf. Section 5); in other words, we consider the rotations of the horospherical wavefronts. The generic situation, however, is that a square-integrable packet is driven away from the limit cycle and starts to spiral toward the boundary, like the classical trajectories. It stays confined to the limit cycle, 
apart from dispersion, only if the covering packet in $H^{3}$ is centered at $C(\Lambda)$, the convex hull of the limit set.

Equation (5.24) with the $f_{m=0}$ in (6.4) is solved by $\Delta \tau \sim \tau(|K|-1)$. The package goes on rotating forever in linearly increasing periods, corresponding to the increase of the circumference of the limit cycle. If $m \neq 0$ and $\tau$ is sufficiently large, then equation (5.24) has no positive $\Delta \tau$ as solution; after a finite number of rotations the particle comes to rest.

In the early stage of the expansion, $a(\tau) \sim(\Lambda \tau)^{\lambda}, \tau \rightarrow 0, \lambda>0$ [cf. (5.15)], with the phase $f$ given in Tomaschitz (1993a) [cf. Eqs. (5.28), (5.36)], we have

$$
\Delta \tau \sim \frac{1}{\Lambda} \frac{\log |K|}{s_{0}}\left(s_{0}^{2}+1-6 \xi\right)^{1 / 2}(\Lambda \tau)^{\lambda}
$$

and the packet is rotating independent of its mass with a frequency

$$
v \sim \frac{\Lambda\left(s_{0}^{2}+1-6 \xi\right)^{1 / 2}}{2 \pi}(\Lambda \tau)^{-\lambda} \rightarrow \infty
$$

and a group velocity $c s_{0} /\left(s_{0}^{2}+1-6 \xi\right)^{1 / 2}, \xi \leq 1 / 6$.

We have for both massive and massless particles, if $\xi \neq 1 / 6$,

$$
(\chi / d) \sim \text { const } \cdot \tau^{1-\lambda} \rightarrow \infty
$$

and if $\xi=1 / 6$,

$$
\frac{\chi}{d}=\frac{1}{\alpha \log |K|}\left[1+\frac{\alpha^{4}}{2}\left(\frac{m c^{2}}{\hbar \Lambda}\right)^{4} \frac{(\Lambda \tau)^{2(1+\lambda)}}{(1+\lambda)^{2} s_{0}^{6}}+O\left(\tau^{4(1+\lambda)}\right)\right]
$$

Thus, if the field is not conformally coupled, it disperses, and we have self-interference, as described in Section 5 . In the conformally coupled case the packet has again an intrinsic finite limit width, so that it cannot overlap with itself on the limit cycle. Analogous considerations concerning dispersion hold true for the classical density (5.1), because of (5.23).

Clearly it would be interesting to compare the size of the limit cycle to atomic length scales. In order to do so we would have to know the point in the deformation space [the $K$ in Eq. (6.1)] that determines the metric at present. Equations that determine the evolution of the metric in the deformation space are still lacking.

In a generic infinite 3-space of higher connectivity, the convex hull of the limit set is a three-dimensional finite domain. Its diameter defines then a length scale whose actual size is again determined by the choice of the metric in the deformation space of the 3-manifold. The chaoticity of the classical trajectories in the convex hull could be an explanation for the uniform distribution of galaxies. 
Finally, topological self-interference leads to parity violation in the free Dirac equation on a multiply connected RW background (Tomaschitz, 1994). A $P$-reflected wave packet can cover the limit cycle and overlap with itself. This causes self-interference, its norm is not preserved, and accordingly $P$ is not a unitary symmetry. The $C P T$ symmetry is likewise broken.

\section{ACKNOWLEDGMENTS}

The author acknowledges the support of the European Communities through their Science Programme under grant $\mathrm{B} / \mathrm{SCl}^{*} \mathbf{-}_{-915078 .}$

I also express my sincere thanks to Vinayak Wadke for the accurate drawings.

\section{REFERENCES}

Akaza, T. (1964). Nagoya Mathematical Journal, 24, 43-64.

Beardon, A. (1983). The Geometry of Discrete Groups, Springer, Berlin.

Bers, L. (1970). Annals of Mathematics, 91, 570-600.

Ford, L. (1951). Automorphic Functions, Chelsea, New York.

Krushkal, S. L., Apanasov, B. N., and Grusevskii, N. A. (1986). Kleinian Groups and Uniformization in Examples and Problems, American Mathematical Society, Providence, Rhode Island.

Landau, L. D., and Lifshitz, E. M. (1971). The Classical Theory of Fields, Pergamon, London. Mandouvalos, N. (1988). Proceedings of the London Mathematical Society, 57, 209-238.

Patterson, S. J. (1987). Lectures on measures on limit sets of Kleinian groups, in Analytical and Geometrical Aspects of Hyperbolic Space, D. B. A. Epstein, ed., Cambridge University Press, Cambridge, pp. 281-323.

Schrödinger, E. (1939). Physica, 6, 899-912.

Schrödinger, E. (1956). Expanding Universes, Cambridge University Press, Cambridge.

Tomaschitz, R. (1991). Journal of Mathematical Physics, 32, 2571-2579.

Tomaschitz, R. (1992a). International Journal of Theoretical Physics, 31, 187-210.

Tomaschitz, R. (1992b). Complex Systems 6, 137-161.

Tomaschitz, R. (1992c). Chaotic dynamics in general relativity, in Chaotic Dynamics: Theory and Practice, T. Bountis, ed., Plenum Press, New York, pp. 122-139.

Tomaschitz, R. (1993a). Journal of Mathematical Physics, 34, 1022-1042.

Tomaschitz, R. (1993b). Journal of Mathematical Physics, 34, 3133-3150.

Tomaschitz, R. (1994a). Classical and quantum chaos in extended RW cosmologies, in Deterministic Chaos in General Relativity, D. Hobill, ed., Plenum Press, New York, to appear.

Tomaschitz, R. (1994b). Cosmological CP violation, Journal of Mathematical Physics, 35, to appear.

Wheeler, J. A. (1973). From relativity to mutability, in The Physicist's Conception of Nature, J. Mehra, ed., Reidel, Dordrecht, pp. 202-247.

Whittaker, E. T., and Watson, G. N. (1984). A Course of Modern Analysis, Cambridge University Press, Cambridge. 